\title{
Necessary and sufficient conditions for boundedness of the maximal operator in local Morrey-type spaces
}

\author{
by \\ Viktor I. Burenkov and Huseyn V. Guliyev (Cardiff)
}

\begin{abstract}
The problem of boundedness of the Hardy-Littewood maximal operator in local and global Morrey-type spaces is reduced to the problem of boundedness of the Hardy operator in weighted $L_{p}$-spaces on the cone of non-negative non-increasing functions. This allows obtaining sufficient conditions for boundedness for all admissible values of the parameters. Moreover, in case of local Morrey-type spaces, for some values of the parameters, these sufficient conditions are also necessary.
\end{abstract}

For $x \in \mathbb{R}^{n}$ and $r>0$, let $B(x, r)$ denote the open ball centred at $x$ of radius $r$.

Definition 1. Let $0<p, \theta \leq \infty$ and let $w$ be a non-negative measurable function on $(0, \infty)$. We denote by $L M_{p \theta, w}$ and $G M_{p \theta, w}$ the local and global Morrey-type spaces respectively, defined to be the spaces of all functions $f \in L_{p}^{\operatorname{loc}}\left(\mathbb{R}^{n}\right)$ with finite quasinorms

$$
\begin{aligned}
\|f\|_{L M_{p \theta, w}} & \equiv\|f\|_{L M_{p \theta, w}\left(\mathbb{R}^{n}\right)}=\|w(r)\| f\left\|_{L_{p}(B(0, r))}\right\|_{L_{\theta}(0, \infty)}, \\
\|f\|_{G M_{p \theta, w}} & =\sup _{x \in \mathbb{R}^{n}}\|f(x+\cdot)\|_{L M_{p \theta, w}}
\end{aligned}
$$

respectively.

Lemma 1. Let $0<p, \theta \leq \infty$ and let $w$ be a non-negative measurable function on $(0, \infty)$.

(i) If

$$
\|w(r)\|_{L_{\theta}(t, \infty)}=\infty \quad \text { for all } t>0,
$$

then $L M_{p \theta, w}=G M_{p \theta, w}=\Theta$, where $\Theta$ is the set of all functions equivalent to 0 on $\mathbb{R}^{n}$.

(ii) If

$$
\left\|w(r) r^{n / p}\right\|_{L_{\theta}(0, t)}=\infty \quad \text { for all } t>0,
$$

2000 Mathematics Subject Classification: Primary 42B25.

Supported by the grants of Russian Foundation for Basic Research (project 02-0100602) and NATO (project PST.CLG.978412). 
then $f(0)=0$ for all $f \in L M_{p \theta, w}$ continuous at 0 , and $G M_{p \theta, w}=\Theta$ for $0<p<\infty$.

Proof. (i) Let (1) be satisfied and $f$ be not equivalent to zero. Then $A=\|f\|_{L_{p}\left(B\left(0, t_{0}\right)\right)}>0$ for some $t_{0}>0$. Hence

$$
\|f\|_{G M_{p \theta, w}} \geq\|f\|_{L M_{p \theta, w}} \geq\|w(r)\| f\left\|_{L_{p}(B(0, r))}\right\|_{L_{\theta}\left(t_{0}, \infty\right)} \geq A\|w(r)\|_{L_{\theta}\left(t_{0}, \infty\right)} .
$$

Therefore $\|f\|_{G M_{p \theta, w}}=\|f\|_{L M_{p \theta, w}}=\infty$.

(ii) Let (2) be satisfied. If $f \in L M_{p \theta, w}$ and there exists

$$
\lim _{r \rightarrow 0}|B(0, r)|^{-1 / p}\|f\|_{L_{p}(B(0, r))}=B
$$

where $|B(0, r)|$ is the volume of $B(0, r)$, then $B=0$. Indeed, assume $B>0$. Then there exists $t_{0}>0$ such that

$$
|B(0, r)|^{-1 / p}\|f\|_{L_{p}(B(0, r))} \geq B / 2
$$

for all $0<r \leq t_{0}$. Consequently,

$$
\|f\|_{L M_{p \theta, w}} \geq\|w(r)\| f\left\|_{L_{p}(B(0, r))}\right\|_{L_{\theta}\left(0, t_{0}\right)} \geq \frac{B}{2} v_{n}^{1 / p}\left\|w(r) r^{n / p}\right\|_{L_{\theta}\left(0, t_{0}\right)},
$$

where $v_{n}$ is the volume of the unit ball in $\mathbb{R}^{n}$. Hence $\|f\|_{L M_{p \theta, w}}=\infty$, so $f \notin L M_{p \theta, w}$ and we have arrived at a contradiction.

If $f \in L M_{p \theta, w}$ and it is continuous at 0 , then (3) holds with $B=|f(0)|$. Hence $f(0)=0$.

Next let $0<p<\infty$ and let $f \in G M_{p \theta, w}$. Then by the Lebesgue theorem on differentiation of integrals, for almost all $x \in \mathbb{R}^{n}$,

$$
\lim _{r \rightarrow 0}|B(x, r)|^{-1 / p}\|f\|_{L_{p}(B(x, r))}=|f(x)| .
$$

By the above argument, $f(x)=0$ for all those $x$. Hence $f$ is equivalent to zero.

Definition 2. Let $0<p, \theta \leq \infty$. We denote by $\Omega_{p, \theta}$ the set of all nonnegative measurable functions $w$ on $(0, \infty)$ such that for some $t_{1}, t_{2}>0$,

$$
\|w(r)\|_{L_{\theta}\left(t_{1}, \infty\right)}<\infty, \quad\left\|w(r) r^{n / p}\right\|_{L_{\theta}\left(0, t_{2}\right)}<\infty .
$$

In what follows, keeping in mind Lemma 1 , we always assume that $w \in$ $\Omega_{p, \theta}$.

The spaces $G M_{p \infty, r^{-\lambda / p}}$, where $0<\lambda<n$, are the classical Morrey spaces introduced in [6] and applied to studying the local behaviour of solutions of second order elliptic partial differential equations. The interpolation properties of the spaces $G M_{p \infty, w}$ were investigated by S. Spanne in [11]. The spaces $G M_{p \theta, r^{-\lambda}}$ were used by G. Lu [4] for studying embedding theorems for vector fields of Hörmander type. T. Mizuhara [5] and E. Nakai [7] studied the boundedness of various integral operators in the spaces $G M_{p \infty, w}$.

For a measurable set $\Omega \subset \mathbb{R}^{n}$ and a non-negative measurable function $v$ on $\Omega$, let $L_{p, v}(\Omega)$ be the weighted $L_{p^{-}}$space of all measurable functions $f$ 
on $\Omega$ for which

$$
\|f\|_{L_{p, v}(\Omega)}=\|v f\|_{L_{p}(\Omega)}<\infty .
$$

If $0<p \leq \theta \leq \infty$, then

$$
\|f\|_{L M_{p \theta, w}} \leq\|f\|_{L_{p, W}\left(\mathbb{R}^{n}\right)}
$$

and if $0<\theta \leq p \leq \infty$, then

$$
\|f\|_{L_{p, W}\left(\mathbb{R}^{n}\right)} \leq\|f\|_{L M_{p \theta, w}}
$$

where

$$
W(x)=\|w\|_{L_{\theta}(|x|, \infty)} \quad \text { for } x \in \mathbb{R}^{n} .
$$

These inequalities are particular cases of general inequalities for Lebesgue spaces with mixed quasinorms (see, for example, [8, Section 3.37]). In particular, for $0<p \leq \infty$,

$$
\|f\|_{L M_{p p, w}}=\|f\|_{L_{p, W_{p}}\left(\mathbb{R}^{n}\right)} .
$$

where $W_{p}(x)=\|w\|_{L_{p}(|x|, \infty)}$ for $x \in \mathbb{R}^{n}$.

Let $f \in L_{1}^{\text {loc }}\left(\mathbb{R}^{n}\right)$. The Hardy-Littlewood maximal operator $M$ is defined by

$$
(M f)(x)=\sup _{r>0}|B(x, r)|^{-1} \int_{B(x, r)}|f(y)| d y .
$$

F. Chiarenza and M. Frasca [2] have proved the boundedness of $M$ in the classical Morrey spaces. T. Mizuhara [5] and E. Nakai [7] have obtained sufficient conditions on $w$ for the boundedness of $M$ in $G M_{p \infty, w}$. In this paper we improve, in particular, the results obtained in [5, 7]. Moreover, for some values of parameters we obtain necessary and sufficient conditions for the operator $M$ to be bounded from $L M_{p \theta_{1}, w_{1}}$ to $L M_{p \theta_{2}, w_{2}}$.

An application of the known necessary and sufficient conditions for the boundedness of the maximal operator from one weighted Lebesgue space to another [10] immediately implies the following result for local Morrey-type spaces. Here and throughout, $\chi_{E}$ denotes the characteristic function of the set $E$.

ThEOREM 1. Let $1<p_{1} \leq p_{2}<\infty, 0<\theta_{1}, \theta_{2} \leq \infty, w_{1} \in \Omega_{p_{1}, \theta_{1}}$, $w_{2} \in \Omega_{p_{2}, \theta_{2}}$.

- If $p_{1} \geq \theta_{1}$ and $p_{2} \leq \theta_{2}$, and for some $c_{1}>0$ and all balls $B \subset \mathbb{R}^{n}$,

$$
\left\|M\left(\chi_{B} W_{1}^{p_{1} /\left(1-p_{1}\right)}\right)\right\|_{L_{p_{2}, W_{2}}(B)} \leq c_{1}\left\|W_{1}^{1 /\left(1-p_{1}\right)}\right\|_{L_{p_{1}}(B)}
$$

where

$$
W_{1}(x)=\left\|w_{1}\right\|_{L_{\theta_{1}}(|x|, \infty)}, \quad W_{2}(x)=\left\|w_{2}\right\|_{L_{\theta_{2}}(|x|, \infty)}, \quad x \in \mathbb{R}^{n},
$$

then the operator $M$ is bounded from $L M_{p_{1} \theta_{1}, w_{1}}$ to $L M_{p_{2} \theta_{2}, w_{2}}$ and from $G M_{p_{1} \theta_{1}, w_{1}}$ to $G M_{p_{2} \theta_{2}, w_{2}}$. 
- If $p_{1} \leq \theta_{1}$ and $p_{2} \geq \theta_{2}$, then condition (8) is necessary for the boundedness of $M$ from $L M_{p_{1} \theta_{1}, w_{1}}$ to $L M_{p_{2} \theta_{2}, w_{2}}$.

- In particular, if $\theta_{1}=p_{1}$ and $\theta_{2}=p_{2}$, then condition (8) is necessary and sufficient for the boundedness of $M$ from $L M_{p_{1} p_{1}, w_{1}}$ to $L M_{p_{2} p_{2}, w_{2}}$.

Proof. Let $p_{1} \geq \theta_{1}$ and $p_{2} \leq \theta_{2}$. By applying (6), the sufficiency of (8) for the boundedness of $M$ and (7) we get

$$
\begin{aligned}
\|M f\|_{L M_{p_{2} \theta_{2}, w_{2}}} & \leq\|M f\|_{L_{p_{2}, W_{2}}\left(\mathbb{R}^{n}\right)} \\
& \leq c_{2}\|f\|_{L_{p_{1}, W_{1}}\left(\mathbb{R}^{n}\right)} \leq c_{2}\|f\|_{L M_{p_{1} \theta_{1}, w_{1}}},
\end{aligned}
$$

where $c_{2}>0$ is independent of $f$.

Conversely, if $p_{1} \leq \theta_{1}, p_{2} \geq \theta_{2}$, and

$$
\|M f\|_{L M_{p_{2} \theta_{2}, w_{2}}} \leq c_{3}\|f\|_{L M_{p_{1} \theta_{1}, w_{1}}}
$$

where $c_{3}>0$ is independent of $f$, then by (6),

$$
\|M f\|_{L_{p_{2}, W_{2}}\left(\mathbb{R}^{n}\right)} \leq c_{3}\|f\|_{L_{p_{1}, W_{1}}\left(\mathbb{R}^{n}\right)}
$$

which is known to imply (8).

Also (9) implies that

$$
\|M f\|_{G M_{p_{2} \theta_{2}, w_{2}}} \leq c_{2}\|f\|_{G M_{p_{1} \theta_{1}, w_{1}}} .
$$

In order to obtain conditions on $w_{1}$ and $w_{2}$ ensuring the boundedness of $M$ for other values of the parameters and to obtain simpler conditions for $p=\theta_{1}=\theta_{2}$ we shall reduce the problem of boundedness of $M$ in local Morrey-type spaces to the problem of boundedness of Hardy operator in weighted $L_{p}$-spaces on the cone of non-negative non-increasing functions.

As in $[2,5,7]$, we start with the inequality

$$
\int_{B(0, r)}(M f)(x)^{p} d x \leq c_{4} \int_{\mathbb{R}^{n}}|f(x)|^{p}\left(M \chi_{B(0, r)}\right)(x) d x,
$$

where $1<p<\infty$ and $c_{4}>0$ is independent of $f \in L_{1}^{\text {loc }}\left(\mathbb{R}^{n}\right)$ and $r$. This is a particular case of a more general inequality established by C. L. Fefferman and E. Stein [3]:

$$
\int_{\mathbb{R}^{n}}(M f)(x)^{p}|\varphi(x)| d x \leq c_{5} \int_{\mathbb{R}^{n}}|f(x)|^{p}(M \varphi)(x) d x,
$$

where $1<p<\infty$ and $c_{5}>0$ is independent of $f, \varphi \in L_{1}^{\text {loc }}\left(\mathbb{R}^{n}\right)$.

Lemma 2. For all $r>0$ and $x \in \mathbb{R}^{n}$,

$$
\left(\frac{r}{|x|+r}\right)^{n} \leq\left(M \chi_{B(0, r)}\right)(x) \leq 4^{n}\left(\frac{r}{|x|+r}\right)^{n} .
$$

This statement is known, at least with some constant on the right-hand side. For the sake of completeness we give the proof. 
Proof. If $n=1$, then $M \chi_{(-r, r)}=1$ for $|x|<r$ and $M \chi_{(-r, r)}=r /(|x|+r)$ if $|x| \geq r$, and (12) is trivial.

Assume that $n \geq 2$. If $|x|<r$, then $M \chi_{B(0, r)}=1$ and (12) is trivial again.

Assume that $|x| \geq r$. If $\varrho \geq|x|$, then

$$
\begin{aligned}
G(x, r, \varrho) & =\frac{1}{|B(x, \varrho)|} \int_{B(x, \varrho)} \chi_{B(0, r)}(y) d y=\frac{|B(x, \varrho) \cap B(0, r)|}{|B(x, \varrho)|} \\
& \leq\left(\frac{r}{\varrho}\right)^{n} \leq\left(\frac{r}{|x|}\right)^{n} .
\end{aligned}
$$

If $0<\varrho \leq|x|-r$, then $G(x, r, \varrho)=0$.

Assume that $|x|-r \leq \varrho \leq|x|$. In order to estimate $G(x, r, \varrho)$ from above note that

$$
B(x, \varrho) \cap B(0, r) \subset B_{n-1}(0, h) \times(|x|-\varrho, r),
$$

where $B_{n-1}(0, h)$ is the ball centered at the origin of radius $h$ in $\mathbb{R}^{n-1}$, and $h$ is the height in the triangle with side lengths $r, \varrho$ and $|x|$, perpendicular to the side of length $|x|$. Since

$$
h=\frac{\sqrt{\varrho^{2}-(|x|-r)^{2}} \sqrt{(|x|+r)^{2}-\varrho^{2}}}{2|x|} \leq \sqrt{\left(\varrho^{2}-(|x|-r)^{2}\right) \frac{r}{|x|}},
$$

it follows that

$$
\begin{aligned}
G(x, r, \varrho) & \leq \frac{v_{n-1}}{v_{n}} \frac{\left(\left(\varrho^{2}-(|x|-r)^{2}\right) r /|x|\right)^{(n-1) / 2}(\varrho-(|x|-r))}{\varrho^{n}} \\
& =\frac{v_{n-1}}{v_{n}}\left(\left(1-\left(\frac{|x|-r}{\varrho}\right)^{2}\right) \frac{r}{|x|}\right)^{(n-1) / 2}\left(1-\frac{|x|-r}{\varrho}\right) \\
& \leq \frac{v_{n-1}}{v_{n}}\left(\left(1-\left(\frac{|x|-r}{|x|}\right)^{2}\right) \frac{r}{|x|}\right)^{(n-1) / 2}\left(1-\frac{|x|-r}{|x|}\right) \\
& \leq \frac{v_{n-1}}{v_{n}} 2^{(n-1) / 2}\left(\frac{r}{|x|}\right)^{n} .
\end{aligned}
$$

Since $v_{n-1} / v_{n} \leq n / 2 \leq 2^{n / 2}$, for $|x| \geq r$ and for all $\varrho \geq|x|-r$ we have

$$
G(x, r, \varrho) \leq 2^{n}\left(\frac{r}{|x|}\right)^{n} \leq 4^{n}\left(\frac{r}{|x|+r}\right)^{n} .
$$

Since

$$
M \chi_{B(0, r)}(x)=\sup _{\varrho \geq|x|-r} G(x, r, \varrho),
$$

the upper bound in (12) follows. The lower bound is much simpler:

$$
M \chi_{B(0, r)}(x) \geq G(x, r,|x|+r)=\left(\frac{r}{|x|+r}\right)^{n}
$$


Corollary 1. Let $1<p<\infty$. Then there exists $c_{6}>0$ such that

$$
\|M f\|_{L_{p}(B(0, r))} \leq c_{6}\left(r^{n} \int_{\mathbb{R}^{n}} \frac{|f(x)|^{p}}{(|x|+r)^{n}} d x\right)^{1 / p},
$$

for all $r>0$ and all $f \in L_{1}^{\text {loc }}\left(\mathbb{R}^{n}\right)$.

In order to estimate the right-hand side integral we first prove the following equality.

Lemma 3. Let $\phi$ be a non-negative measurable function on $\mathbb{R}^{n}$. Then for all $r \geq 0$,

$$
\int_{|x| \geq r} \frac{\phi(x) d x}{|x|^{n}}=n \int_{r}^{\infty}\left(\int_{r \leq|x| \leq t} \phi(x) d x\right) \frac{d t}{t^{n+1}} .
$$

Proof. By applying spherical coordinates we get

$$
\begin{aligned}
\int_{r}^{\infty}\left(\int_{r \leq|x| \leq t} \phi(x) d x\right) \frac{d t}{t^{n+1}} & =\int_{r}^{\infty}\left(\int_{S^{n-1}}\left(\int_{r}^{t} \phi(\varrho \sigma) \varrho^{n-1} d \varrho\right) d \sigma\right) \frac{d t}{t^{n+1}} \\
& =\int_{S^{n-1}}\left(\int_{r}^{\infty}\left(\int_{\max \{\varrho, r\}}^{\infty} \frac{d t}{t^{n+1}}\right) \phi(\varrho \sigma) \varrho^{n-1} d \varrho\right) d \sigma \\
& =\frac{1}{n} \int_{S^{n-1}} \int_{r}^{\infty} \frac{\phi(\varrho \sigma) \varrho^{n-1}}{\varrho^{n}} d \varrho d \sigma=\frac{1}{n} \int_{|x| \geq r} \frac{\phi(x) d x}{|x|^{n}}
\end{aligned}
$$

LEMma 4. Let $\varphi$ be a non-negative measurable function on $\mathbb{R}^{n}$. Then for all $r \geq 0$,

$$
n 2^{-n} \int_{r}^{\infty}\left(\int_{B(0, t)} \varphi(x) d x\right) \frac{d t}{t^{n+1}} \leq \int_{\mathbb{R}^{n}} \frac{\varphi(x) d x}{(|x|+r)^{n}} \leq n \int_{r}^{\infty}\left(\int_{B(0, t)} \varphi(x) d x\right) \frac{d t}{t^{n+1}} .
$$

Proof. Since

$$
\int_{\mathbb{R}^{n}} \frac{\varphi(x) d x}{(|x|+r)^{n}} \leq r^{-n} \int_{|x| \leq r} \varphi(x) d x+\int_{|x|>r} \frac{\varphi(x)}{|x|^{n}} d x
$$

and

$$
\int_{\mathbb{R}^{n}} \frac{\varphi(x) d x}{(|x|+r)^{n}} \geq 2^{-n}\left(r^{-n} \int_{|x| \leq r} \varphi(x) d x+\int_{|x|>r} \frac{\varphi(x)}{|x|^{n}} d x\right),
$$

the statement follows by Lemma 3 because

$$
\begin{aligned}
\int_{|x| \geq r} \frac{\varphi(x)}{|x|^{n}} d x & =n \int_{r}^{\infty}\left(\int_{|x| \leq t} \varphi(x) d x-\int_{|x| \leq r} \varphi(x) d x\right) \frac{d t}{t^{n+1}} \\
& =n \int_{r}^{\infty}\left(\int_{|x| \leq t} \varphi(x) d x\right) \frac{d t}{t^{n+1}}-r^{-n} \int_{|x| \leq r} \varphi(x) d x
\end{aligned}
$$


Corollary 2. Let $1<p<\infty$. Then there exists $c_{7}>0$ such that

$$
\|M f\|_{L_{p}(B(0, r))} \leq c_{7}\left(r^{n} \int_{r}^{\infty}\left(\int_{B(0, t)}|f(x)|^{p} d x\right) \frac{d t}{t^{n+1}}\right)^{1 / p}
$$

for all $r>0$ and all $f \in L_{1}^{\text {loc }}\left(\mathbb{R}^{n}\right)$.

Let $H$ be the Hardy operator:

$$
(H g)(r)=\int_{0}^{r} g(t) d t, \quad 0<r<\infty .
$$

Lemma 5. Let $1<p<\infty, 0<\theta \leq \infty, w \in \Omega_{p, \theta}$. Then there exists $c_{8}>0$ such that

$$
\|M f\|_{L M_{p \theta, w}} \leq c_{8}\|H g\|_{L_{\theta / p, v}(0, \infty)}^{1 / p}
$$

for all $f \in L_{p}^{\text {loc }}\left(\mathbb{R}^{n}\right)$, where

$$
\begin{aligned}
g(t) & =\int_{B\left(0, t^{-1 / n}\right)}|f(y)|^{p} d y, \\
v(r) & =w\left(r^{-1 / n}\right)^{p} r^{-1-(1+1 / n) p / \theta} .
\end{aligned}
$$

Proof. By Corollary 2, for $\theta<\infty$ we have

$$
\begin{aligned}
& \|M f\|_{L M_{p \theta, w}}=\|w(r)\| M f\left\|_{L_{p}(B(0, r))}\right\|_{L_{\theta}(0, \infty)} \\
& \leq c_{7}^{1 / p}\left\|w(r) r^{n / p}\left(\int_{r}^{\infty}\left(\int_{B(0, t)}|f(x)|^{p} d x\right) \frac{d t}{t^{n+1}}\right)^{1 / p}\right\|_{L_{\theta}(0, \infty)} \\
& =c_{7}^{1 / p} n^{-1 / p}\left\|w(r) r^{n / p}\left(\int_{0}^{r^{-n}}\left(\int_{B\left(0, \tau^{-1 / n}\right)}|f(x)|^{p} d x\right) d \tau\right)^{1 / p}\right\|_{L_{\theta}(0, \infty)} \\
& =c_{7}^{1 / p} n^{-1 / p}\left(\int_{0}^{\infty}\left(w(r) r^{n / p}\right)^{\theta}\left(\int_{0}^{r^{-n}} g(\tau) d \tau\right)^{\theta / p} d r\right)^{1 / \theta} \\
& =c_{7}^{1 / p} n^{-1 / p-1 / \theta}\left(\int_{0}^{\infty}\left(w\left(\varrho^{-1 / n}\right) \varrho^{-1 / p}\right)^{\theta} \varrho^{-1 / n-1}\left(\int_{0}^{\varrho} g(\tau) d \tau\right)^{\theta / p} d \varrho\right)^{1 / \theta} \\
& =c_{8}\|H g\|_{L_{\theta / p, v}(0, \infty)}^{1 / p}
\end{aligned}
$$

If $\theta=\infty$, then by a similar argument

$$
\begin{aligned}
\|M f\|_{L M_{p \infty, w}} & =\underset{0<r<\infty}{\operatorname{ess} \sup } w(r)\|M f\|_{L_{p}(B(0, r))} \\
\leq & c_{8}^{\prime} \underset{0<\varrho<\infty}{\operatorname{ess} \sup } w\left(\varrho^{-1 / n}\right) \varrho^{-1 / p} H g^{1 / p}(\varrho), \quad c_{8}^{\prime}=c_{7}^{-1 / p} n^{-1 / p} .
\end{aligned}
$$


Corollary 3. Let $1<p<\infty, 0<\theta \leq \infty, w \in \Omega_{p, \theta}$. Then

$$
\|M f\|_{G M_{p \theta, w}} \leq c_{8} \sup _{x \in \mathbb{R}^{n}}\|H(g(x, \cdot))\|_{L_{\theta / p, v}(0, \infty)}^{1 / p}
$$

for all $f \in L_{p}^{\text {loc }}\left(\mathbb{R}^{n}\right)$, where $v$ is given by (16) and

$$
g(x, t)=\int_{B\left(x, t^{-1 / n}\right)}|f(y)|^{p} d y=\int_{B\left(0, t^{-1 / n}\right)}|f(x+y)|^{p} d y .
$$

Proof. Since for all $x \in \mathbb{R}^{n}$,

$$
\begin{aligned}
\|(M f)(x+\cdot) & \|_{L_{p}(B(0, r))} \\
& =\left(\int_{B(0, r)}\left(\sup _{r>0} \frac{1}{|B(x+z, r)|} \int_{B(x+z, r)}|f(y)| d y\right)^{p} d z\right)^{1 / p} \\
& =\left(\int_{B(0, r)}\left(\sup _{r>0} \frac{1}{|B(z, r)|} \int_{B(z, r)}|f(x+u)| d u\right)^{p} d z\right)^{1 / p} \\
& =\|M(f(x+\cdot))\|_{L_{p}(B(0, r))},
\end{aligned}
$$

we have

$$
\begin{aligned}
\|M f\|_{G M_{p \theta, w}} & =\sup _{x \in \mathbb{R}^{n}}\|w(r)\|(M f)(x+\cdot)\left\|_{L_{p}(B(0, r))}\right\|_{L_{\theta}(0, \infty)} \\
& =\sup _{x \in \mathbb{R}^{n}}\|w(r)\| M(f(x+\cdot))\left\|_{L_{p}(B(0, r))}\right\|_{L_{\theta}(0, \infty)} \\
& =\sup _{x \in \mathbb{R}^{n}}\|M(f(x+\cdot))\|_{L M_{p \theta, w}} \\
& \leq c_{8} \sup _{x \in \mathbb{R}^{n}}\left\|H\left(\left.\int_{B\left(0, t^{-1 / n}\right)} f(x+y)\right|^{p} d y\right)\right\|_{L_{\theta / p, v}(0, \infty)}^{1 / p} \\
& =c_{8} \sup _{x \in \mathbb{R}^{n}}\|H(g(x, \cdot))\|_{L_{\theta / p, v}(0, \infty)}^{1 / p} .
\end{aligned}
$$

Theorem 2. Let $0<p_{2} \leq p_{1}<\infty, p_{1}>1,0<\theta_{1}, \theta_{2} \leq \infty, w_{1} \in \Omega_{p_{1}, \theta_{1}}$, $w_{2} \in \Omega_{p_{2}, \theta_{2}}$. Assume that, for some $q>1$ satisfying $p_{2} \leq q \leq p_{1}$, the operator $H$ is bounded from $L_{\theta_{1} / q, v_{1}}(0, \infty)$ to $L_{\theta_{2} / q, v_{2}}(0, \infty)$ on the cone of all nonnegative functions $\varphi$ non-increasing on $(0, \infty)$ and satisfying $\lim _{t \rightarrow \infty} \varphi(t)$ $=0$, where

$$
\begin{aligned}
& v_{1}(r)=\left(w_{1}\left(r^{-1 / n}\right) r^{1 / q-1 / p_{1}-(1+1 / n) / \theta_{1}}\right)^{q}, \\
& v_{2}(r)=\left(w_{2}\left(r^{-1 / n}\right) r^{-1 / p_{2}-(1+1 / n) / \theta_{2}}\right)^{q} .
\end{aligned}
$$

Then the operator $M$ is bounded from $L M_{p_{1} \theta_{1}, w_{1}}$ to $L M_{p_{2} \theta_{2}, w_{2}}$ and from $G M_{p_{1} \theta_{1}, w_{1}}$ to $G M_{p_{2} \theta_{2}, w_{2}}$. 
Proof. 1. By Hölder's inequality with exponent $q / p_{2} \geq 1$,

$$
\begin{aligned}
\|M f\|_{L M_{p_{2} \theta_{2}, w_{2}}} & =\left\|w_{2}(r)\right\| M f\left\|_{L_{p_{2}}(B(0, r))}\right\|_{L_{\theta_{2}}(0, \infty)} \\
& \leq\left\|w_{2}(r)|B(0, r)|^{1 / p_{2}-1 / q}\right\| M f\left\|_{L_{q}(B(0, r))}\right\|_{L_{\theta_{2}}(0, \infty)} \\
& =v_{n}^{1 / p_{2}-1 / q}\left\|u_{2}(r)\right\| M f\left\|_{L_{q}(B(0, r))}\right\|_{L_{\theta_{2}}(0, \infty)} \\
& =v_{n}^{1 / p_{2}-1 / q}\|M f\|_{L M_{q \theta_{2}, u_{2}}},
\end{aligned}
$$

where

$$
u_{2}(r)=w_{2}(r) r^{n\left(1 / p_{2}-1 / q\right)} .
$$

Since $q>1$, by Lemma 5 applied to $L M_{q \theta_{2}, u_{2}}$ we have

$$
\|M f\|_{L M_{q \theta_{2}, u_{2}}} \leq c_{9}\|H g\|_{L_{\theta_{2} / q, v_{2}}(0, \infty)}^{1 / q},
$$

where

$$
g(t)=\int_{B\left(0, t^{-1 / n}\right)}|f(y)|^{q} d y,
$$

and $c_{9}>0$ is independent of $f$, because $u_{2}\left(r^{-1 / n}\right)^{q} r^{-1-(1+1 / n) q / \theta_{2}}=v_{2}(r)$.

Since $g$ is non-negative, non-increasing on $(0, \infty)$ and $\lim _{t \rightarrow+\infty} g(t)=0$, and $H$ is bounded from $L_{\theta_{1} / q, v_{1}}(0, \infty)$ to $L_{\theta_{2} / q, v_{2}}(0, \infty)$ on the cone of functions containing $g$, we have

$$
\|M f\|_{L M_{p \theta_{2}, w_{2}}} \leq c_{10}\|g\|_{L_{\theta_{1} / q, v_{1}}(0, \infty)}^{1 / q},
$$

where $c_{10}>0$ is independent of $f$.

Finally, by Hölder's inequality with exponent $p_{1} / q \geq 1$,

$$
g(t) \leq\left|B\left(0, t^{-1 / n}\right)\right|^{1-q / p_{1}}\|f\|_{L_{p_{1}}\left(B\left(0, t^{-1 / n}\right)\right)}^{q} .
$$

Hence

$$
\begin{gathered}
\|M f\|_{L M_{p_{2} \theta_{2}, w_{2}}} \leq c_{11}\left(\int_{0}^{\infty} v_{1}(t)^{\theta_{1} / q} t^{-\left(1-q / p_{1}\right) \theta_{1} / q}\|f\|_{L_{p_{1}}\left(B\left(0, t^{-1 / n}\right)\right)}^{\theta_{1}} d t\right)^{1 / \theta_{1}} \\
=c_{11} n^{1 / \theta_{1}}\left(\int_{0}^{\infty} v_{1}\left(r^{-n}\right)^{\theta_{1} / q} r^{n\left(\theta_{1} / q-\theta_{1} / p_{1}\right)-n-1}\|f\|_{L_{p_{1}}(B(0, r))}^{\theta_{1}} d r\right)^{1 / \theta_{1}} \\
=c_{11} n^{1 / \theta_{1}}\left(\int_{0}^{\infty}\left(w_{1}(r)\|f\|_{L_{p_{1}}(B(0, r))}\right)^{\theta_{1}} d r\right)^{1 / \theta_{1}}=c_{11} n^{1 / \theta_{1}}\|f\|_{L M_{p_{1} \theta_{1}, w_{1}}}
\end{gathered}
$$

where $c_{11}>0$ is independent of $f$.

2. To prove the boundedness of $M$ from $G M_{p_{1} \theta_{1}, w_{1}}$ to $G M_{p_{2} \theta_{2}, w_{2}}$ we apply Corollary 3. Since for all $x \in \mathbb{R}^{n}$ the function $g(x, t)$ defined by (18) is non-negative and non-increasing on $(0, \infty)$ and $\lim _{t \rightarrow \infty} g(x, t)=0$, as in 
Step 1 it follows that

$$
\|M f\|_{G M_{p_{2} \theta_{2}, w_{2}}} \leq c_{10} \sup _{x \in \mathbb{R}^{n}}\|g(x, \cdot)\|_{L_{\theta_{1} / q, v_{1}}(0, \infty)}^{1 / q} \cdot
$$

Since by Hölder's inequality

$$
g(x, t) \leq\left|B\left(0, t^{-1 / n}\right)\right|^{1-q / p_{1}}\|f(x+\cdot)\|_{L_{p_{1}}\left(B\left(0, t^{-1 / n}\right)\right)}^{q},
$$

as in the second part of Step 1 we obtain

$$
\begin{aligned}
\|M f\|_{G M_{p_{2} \theta_{2}, w_{2}}} & \leq c_{11} n^{1 / \theta_{1}} \sup _{x \in \mathbb{R}^{n}}\left(\int_{0}^{\infty} w_{1}(r)\|f(x+\cdot)\|_{L_{p_{1}}(B(0, r))}^{\theta_{1}} d r\right)^{1 / \theta_{1}} \\
& =c_{11} n^{1 / \theta_{1}} \sup _{x \in \mathbb{R}^{n}}\|f(x+\cdot)\|_{L M_{p_{1} \theta_{1}, w_{1}}} \\
& =c_{11} n^{1 / \theta_{1}}\|f\|_{G M_{p_{1} \theta_{1}, w_{1}}} \text {. }
\end{aligned}
$$

In order to obtain explicit sufficient conditions on the weight functions ensuring the boundedness of $M$, we first apply the following simple statement.

Lemma 6. Let $0<\theta \leq \infty$, and let $w$ and $v$ be positive measurable functions on $(0, \infty)$. Then the inequality

$$
\|H g\|_{L_{\theta, w}(0, \infty)} \leq c_{12}\|g\|_{L_{\infty, v}(0, \infty)}
$$

is satisfied for all non-negative functions $g$ with $c_{12}>0$ independent of $g$ if, and only if,

$$
B \equiv\left\|w(r) \int_{0}^{r} \frac{d t}{v(t)}\right\|_{L_{\theta}(0, \infty)}<\infty
$$

Moreover, the minimal value of $c_{12}$ is equal to $B$.

Proof. Necessity. Taking $g=1 / v$ in (26) we obtain (27).

Sufficiency. It suffices to note that

$$
\begin{aligned}
\|H g\|_{L_{\theta, w}(0, \infty)} & =\left\|w(r) \int_{0}^{r} g(t) d t\right\|_{L_{\theta}(0, \infty)} \\
& =\left\|w(r) \int_{0}^{r} \frac{g(t)}{v(t)} v(t) d t\right\|_{L_{\theta}(0, \infty)} \leq B\|g\|_{L_{\infty, v}(0, \infty)} .
\end{aligned}
$$

Applying Lemma 6 to Theorem 2 we obtain the following sufficient condition for the boundedness of the maximal operator in local and global Morrey-type spaces.

Theorem 3. Let $1<p<\infty, 0<\theta_{2} \leq \infty, w_{1} \in \Omega_{p, \infty}, w_{2} \in \Omega_{p, \theta_{2}}$ and suppose that

$$
\left\|w_{2}^{p}(r) r^{n+(n+1) p / \theta_{2}} \int_{r}^{\infty} \frac{1}{w_{1}^{p}(t) t^{n+1}} d t\right\|_{L_{\theta_{2} / p}(0, \infty)}<\infty .
$$


Then the operator $M$ is bounded from $L M_{p \infty, w_{1}}$ to $L M_{p \theta_{2}, w_{2}}$ and from $G M_{p \infty, w_{1}}$ to $G M_{p \theta_{2}, w_{2}}$.

A similar result was obtained by E. Nakai [7] for $w_{1}=w_{2}=w^{-1 / p}(r)$ and $\theta_{2}=\infty$ with an extra condition: there exists $c_{13}>0$ such that $r \leq t \leq$ $2 r \Rightarrow 1 / c_{13} \leq w(t) / w(r) \leq c_{13}$.

Necessary and sufficient conditions for the validity of

$$
\|H \varphi\|_{L_{\theta_{2} / q, v_{2}}(0, \infty)} \leq c_{14}\|\varphi\|_{L_{\theta_{1} / q, v_{1}}(0, \infty)},
$$

where $c_{14}>0$ is independent of $\varphi$, for all non-negative non-increasing functions $\varphi$ are known for most of the cases. For detailed information see [12], [13]. Application of any of those conditions gives sufficient conditions for the boundedness of the maximal operator from $L M_{p_{1} \theta_{1}, w_{1}}$ to $L M_{p_{2} \theta_{2}, w_{2}}$ and from $G M_{p_{1} \theta_{1}, w_{1}}$ to $G M_{p_{2} \theta_{2}, w_{2}}$. However, since the reverse of inequality (11) does not hold (take $f \equiv 1$ ), there is no guarantee that an application of necessary and sufficient conditions on $v_{1}$ and $v_{2}$ ensuring the validity of (30) will imply necessary and sufficient conditions for the boundedness of $M$ from $L M_{p_{1} \theta_{1}, w_{1}}$ to $L M_{p_{2} \theta_{2}, w_{2}}$.

Fortunately for certain values of the parameters this is indeed the case, namely for $1<p<\infty, 0<\theta_{1} \leq \theta_{2}<\infty, \theta_{1} \leq p$.

Note that in this case necessary conditions (which are also sufficient) for the validity of (30) for non-negative non-increasing functions are obtained by taking $\varphi=\chi_{(0, t)}$ with an arbitrary $t>0$.

Since in the proof of Theorem 2 inequality (30) is applied to the function $\varphi=g$, where $g$ is given by (24), it is natural to choose, as test functions, functions $f_{t}, t>0$, for which $\int_{B\left(0, u^{-1 / n}\right)}\left|f_{t}(y)\right|^{q} d y$ is equal or close to $A(t) \chi_{(0, t)}(u), u>0$, where $A(t)$ is independent of $u$. The simplest choice is

$$
f_{t}(y)=\chi_{B(0,2 t) \backslash B(0, t)}(y), \quad y \in \mathbb{R}^{n}, t>0 .
$$

Lemma 7. Let $r, t>0$, and $0<p \leq \infty$. Then

$$
\left\|f_{t}\right\|_{L_{p}(B(0, r))}=0, \quad 0<r \leq t, \quad\left\|f_{t}\right\|_{L_{p}(B(0, r))} \leq c_{15} t^{n / p}, \quad t<r<\infty,
$$

where $c_{15}>0$ depends only on $n$ and $p$.

Proof. The statement follows since for measurable $G, \Omega \subset \mathbb{R}^{n}$,

$$
\left\|\chi_{G}\right\|_{L_{p}(\Omega)}=|G \cap \Omega|^{1 / p} .
$$

Hence $\left\|f_{t}\right\|_{L_{p}(B(0, r))}=|(B(0,2 t) \backslash B(0, t)) \cap B(0, r)|^{1 / p}$.

Lemma 8. For all $t>0$ and $x \in \mathbb{R}^{n}$,

$$
\frac{1}{2}\left(\frac{t}{|x|+t}\right)^{n} \leq\left(M f_{t}\right)(x) \leq 8^{n}\left(\frac{t}{|x|+t}\right)^{n} .
$$


Proof. By setting $r=|x|+2 t$ and noting $B(x,|x|+2 t) \supset B(0,2 t) \backslash B(0, t)$ we get

$$
\begin{aligned}
\left(M f_{t}\right)(x) & =\sup _{r>0} \frac{1}{|B(x, r)|} \int_{B(x, r)} \chi_{B(0,2 t) \backslash B(0, t)}(z) d z \\
& \geq \frac{1}{B(x,|x|+2 t)} \int_{B(x,|x|+2 t)} \chi_{B(0,2 t) \backslash B(0, t)} d z \\
& =\frac{1}{v_{n}} \frac{1}{(|x|+2 t)^{n}} \int_{t<|z|<2 t} d z \\
& =\left(2^{n}-1\right) \frac{t^{n}}{(|x|+2 t)^{n}} \geq\left(1-2^{-n}\right) \frac{t^{n}}{(|x|+t)^{n}} \geq \frac{1}{2} \frac{t^{n}}{(|x|+t)^{n}} .
\end{aligned}
$$

Since $\left|f_{t}\right| \leq \chi_{B(0,2 t)}$, by Lemma 2 we obtain

$$
\left(M f_{t}\right)(x) \leq\left(M \chi_{B(0,2 t)}\right)(x) \leq 4^{n}\left(\frac{2 t}{|x|+2 t}\right)^{n} \leq 8^{n}\left(\frac{t}{|x|+t}\right)^{n} .
$$

For functions $F, G$ defined on $(0, \infty) \times(0, \infty)$ we shall write $F \asymp G$ if there exist $c, c^{\prime}>0$ such that $c F(r, t) \leq G \leq c^{\prime} F(r, t)$ for all $r, t \in(0, \infty)$.

Lemma 9. For all $r, t>0$ and $0<p \leq \infty$,

$$
\left\|M f_{t}\right\|_{L_{p}(B(0, r))} \asymp r^{n / p} \begin{cases}\min \left\{1,(t / r)^{n}\right\}, & 0<p<1, \\ \min \left\{1,(t / r)^{n} \ln (e+r / t)\right\}, & p=1, \\ \min \left\{1,(t / r)^{n / p}\right\}, & 1<p \leq \infty .\end{cases}
$$

Proof. By Lemma 8 we get

$$
\begin{array}{rl}
\left.\left(\frac{1}{2}\right)^{p} t^{n p} \int_{B(0, r)} \frac{1}{(|y|}+t\right)^{n p} & d y \\
\leq & \int_{B(0, r)}\left(M f_{t}\right)^{p}(y) d y \leq 8^{n p} t^{n p} \int_{B(0, r)} \frac{1}{(|y|+t)^{n p}} d y .
\end{array}
$$

Furthermore

$$
\int_{B(0, r)} \frac{1}{(|y|+t)^{n p}} d y=n v_{n} \int_{0}^{r} \frac{\tau^{n-1}}{(\tau+t)^{n p}} d \tau .
$$

If $0<r \leq t$, then

$$
\begin{aligned}
\frac{(2 t)^{-n p} r^{n}}{n} & =(2 t)^{-n p} \int_{0}^{r} \tau^{n-1} d \tau<\int_{0}^{r} \frac{\tau^{n-1}}{(\tau+t)^{n p}} d \tau \\
& \leq t^{-n p} \int_{0}^{r} \tau^{n-1} d \tau=\frac{t^{-n p} r^{n}}{n}
\end{aligned}
$$


Hence

$$
2^{-p(n+1)} r^{n} \leq \frac{1}{v_{n}} \int_{B(0, r)}\left(\left(M f_{t}\right)(y)\right)^{p} d y \leq 8^{n p} r^{n}
$$

If $r>t$ then we consider separately three cases.

1. If $0<p<1$, then by applying (34) with $r=t$ we get

$$
\begin{aligned}
\frac{2^{-n p}}{n} r^{n-n p} & \leq \frac{2^{-n p}}{n}\left(t^{n-n p}+r^{n-n p}-t^{n} r^{-n p}\right)=\frac{2^{-n p}}{n} t^{n-n p}+(2 r)^{-n p} \int_{t}^{r} \tau^{n-1} d \tau \\
& \leq \int_{0}^{r} \frac{\tau^{n-1}}{(\tau+t)^{n p}} d \tau=\int_{0}^{t} \frac{\tau^{n-1}}{(\tau+t)^{n p}} d \tau+\int_{t}^{r} \frac{\tau^{n-1}}{(\tau+t)^{n p}} d \tau \\
& \leq \frac{t^{n-n p}}{n}+\int_{t}^{r} \tau^{n-1-n p} d \tau=\frac{t^{n-n p}}{n}+\frac{r^{n-n p}-t^{n-n p}}{n(1-p)} \leq \frac{r^{n-n p}}{n(1-p)}
\end{aligned}
$$

Hence

$$
2^{-p(n+1)} r^{n-n p} t^{n p} \leq \frac{1}{v_{n}} \int_{B(0, r)}\left(\left(M f_{t}\right)(y)\right)^{p} d y \leq \frac{8^{n p}}{1-p} r^{n-n p} t^{n p} .
$$

2. If $p=1$, then

$$
\begin{aligned}
2^{-n} & \left(\frac{1}{n}+\ln \frac{r}{t}\right)=(2 t)^{-n} \int_{0}^{t} \tau^{n-1} d \tau+2^{-n} \int_{t}^{r} \frac{d \tau}{\tau} \leq \int_{0}^{r} \frac{\tau^{n-1}}{(\tau+t)^{n}} d \tau \\
\quad= & \int_{0}^{t} \frac{\tau^{n-1}}{(\tau+t)^{n}} d \tau+\int_{t}^{r} \frac{\tau^{n-1}}{(\tau+t)^{n}} d \tau \leq t^{-n} \int_{0}^{t} \tau^{n-1} d \tau+\int_{t}^{r} \frac{d \tau}{\tau}=\frac{1}{n}+\ln \frac{r}{t} .
\end{aligned}
$$

Hence

$$
2^{-(n+p)}\left(1+n \ln \frac{r}{t}\right) t^{n} \leq \frac{1}{v_{n}} \int_{B(0, r)}\left(M f_{t}\right)(y) d y \leq 8^{n p}\left(1+n \ln \frac{r}{t}\right) t^{n}
$$

3. Finally, if $1<p<\infty$, then

$$
\begin{aligned}
2^{-n p \frac{t^{n-n p}}{n}} & \leq \int_{0}^{t} \frac{\tau^{n-1}}{(\tau+t)^{n p}} d \tau \leq \int_{0}^{r} \frac{\tau^{n-1}}{(\tau+t)^{n p}} d \tau \\
& =\int_{0}^{t} \frac{\tau^{n-1}}{(\tau+t)^{n p}} d \tau+\int_{t}^{r} \frac{\tau^{n-1}}{(\tau+t)^{n p}} d \tau \\
& \leq \frac{t^{n-n p}}{n}+\int_{t}^{\infty} \tau^{n-1-n p} d \tau=\frac{p}{p-1} \frac{t^{n-n p}}{n} .
\end{aligned}
$$


Hence

$$
2^{-p(n+1)} t^{n} \leq \frac{1}{v_{n}} \int_{B(0, r)}\left(\left(M f_{t}\right)(y)\right)^{p} d y \leq 8^{n p} \frac{p}{p-1} t^{n} .
$$

These estimates imply the statement of the lemma.

Corollary 4. For $0<p \leq \infty, p \neq 1$,

$$
\left\|M f_{t}\right\|_{L_{p}(B(0, r))} \asymp\left(\frac{t}{r+t}\right)^{n \min \{1,1 / p\}} r^{n / p} .
$$

Theorem 4. Let $1<p<\infty, 0<\theta_{1}, \theta_{2} \leq \infty, w_{1} \in \Omega_{p, \theta_{1}}, w_{2} \in \Omega_{p, \theta_{2}}$.

- If $\theta_{1} \leq \theta_{2}$, and $\theta_{1} \leq p$ and for some $c_{16}>0$ and all $t>0$,

$$
\left\|w_{2}(r)\left(\frac{r}{t+r}\right)^{n / p}\right\|_{L_{\theta_{2}}(0, \infty)} \leq c_{16}\left\|w_{1}\right\|_{L_{\theta_{1}}(t, \infty)},
$$

then $M$ is bounded from $L M_{p \theta_{1}, w_{1}}$ to $L M_{p \theta_{2}, w_{2}}$ and from $G M_{p \theta_{1}, w_{1}}$ to $G M_{p \theta_{2}, w_{2}}$.

- For any $0<\theta_{1}, \theta_{2} \leq \infty$ condition (35) is necessary for the boundedness of $M$ from $L M_{p \theta_{1}, w_{1}}$ to $L M_{p \theta_{2}, w_{2}}$.

- In particular, if $\theta_{1} \leq \theta_{2}, \theta_{1} \leq p$, then condition (35) is necessary and sufficient for the boundedness of $M$ from $L M_{p \theta_{1}, w_{1}}$ to $L M_{p \theta_{2}, w_{2}}$.

Proof. Sufficiency. It is known [13] that a necessary and sufficient condition for the validity of (30) with $q=p$ for all non-negative decreasing functions $\varphi$ on $(0, \infty)$ has the form: for some $c_{17}>0$ and all $t>0$,

$$
\left\|v_{2}(r) \min \{t, r\}\right\|_{L_{\theta_{2} / p}(0, \infty)} \leq c_{17}\left\|v_{1}(r)\right\|_{L_{\theta_{1} / p}(0, t)} .
$$

Applying this condition to the functions $v_{1}$ and $v_{2}$ given by (21) and (22) we obtain (35).

Indeed, taking into account (21) and (22) and replacing $r^{-1 / n}$ by $\varrho$ and $t^{-1 / n}$ by $\tau$, we get

$$
\left\|w_{2}(\varrho) \varrho^{n / p} \min \left\{\tau^{-n / p}, \varrho^{-n / p}\right\}\right\|_{L_{\theta_{2}}(0, \infty)} \leq c_{18}\left\|w_{1}\right\|_{L_{\theta_{1}}(\tau, \infty)},
$$

where $c_{18}>0$ is independent of $\tau>0$. Hence (35) follows since

$$
\varrho^{n / p} \min \left\{\tau^{-n / p}, \varrho^{-n / p}\right\} \asymp\left(\frac{\varrho}{\varrho+\tau}\right)^{n / p} .
$$

Necessity. Assume that, for some $c_{19}>0$ and all $f \in L M_{p \theta_{1}, w_{1}}$,

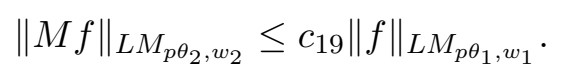

Take $f=f_{t}$, where $f_{t}$ is defined by (31). Then by Lemma 7 the right-hand side of (37) does not exceed

$$
c_{15} t^{n / p}\left\|w_{1}\right\|_{L_{\theta_{1}}(t, \infty)},
$$


where $c_{15}>0$ is independent of $t>0$. Furthermore by Corollary 4 (case $p>1)$ the left-hand side of (37) is equivalent to

$$
\left\|w_{2}(r)\left(\frac{r t}{t+r}\right)^{n / p}\right\|_{L_{\theta_{2}}(0, \infty)}
$$

Hence (35) follows.

REMARK 1. It is unclear whether for $1<p<\infty, \theta_{1} \leq \theta_{2}, \theta_{1} \leq p$ condition (35) is necessary for the boundedness of $M$ from $G M_{p \theta_{1}, w_{1}}$ to $G M_{p \theta_{2}, w_{2}}$. (If we take $f=f_{t}$ in (37), with $L M$ replaced by $G M$, then (35) does not follow.)

REMARK 2. If $p=1,0<\theta_{1}, \theta_{2} \leq \infty$, then a similar argument shows that the condition: there exists $c_{20}>0$ such that for all $t>0$,

$$
\left\|w_{2}(r)\left(\frac{r}{t+r}\right)^{n} \ln \left(e+\frac{r}{t}\right)\right\|_{L_{\theta_{2}}(0, \infty)} \leq c_{20}\left\|w_{1}\right\|_{L_{\theta_{1}}(t, \infty)},
$$

is necessary for the boundedness of $M$ from $L M_{1 \theta_{1}, w_{1}}$ to $L M_{1 \theta_{2}, w_{2}}$.

REMARK 3. Under the assumptions of Theorem 4 the boundedness of the maximal operator from $L M_{p \theta_{1}, w_{1}}$ to $L M_{p \theta_{2}, w_{2}}$ is equivalent to the boundedness of the Hardy operator from $L_{\theta_{1} / p, v_{1}}(0, \infty)$ to $L_{\theta_{2} / p, v_{2}}(0, \infty)$ where $v_{1}(r)=\left(w_{1}\left(r^{-1 / n}\right) r^{-(1+1 / n) 1 / \theta_{1}}\right)^{p}, v_{2}(r)=\left(w_{2}\left(r^{-1 / n}\right) r^{-1 / p-(1+1 / n) 1 / \theta_{2}}\right)^{p}$ on the cone of non-negative non-increasing functions. This is proved by finding necessary and sufficient conditions on $w_{1}$ and $w_{2}$, namely (35), for the boundedness of both operators. It may be of interest to find a direct proof of this equivalence. (One of the implications is established in Theorem 2.)

Next we consider the local and global weak Morrey-type spaces and study the boundedness of the maximal operator $M$ in these spaces.

Definition 3. Let $0<p, \theta \leq \infty$ and let $w$ be a non-negative measurable function on $(0, \infty)$. Denote by $L W M_{p \theta, w}$ and $G W M_{p \theta, w}$ the local and global weak Morrey-type spaces respectively, defined to be the spaces of all functions $f \in W L_{p}^{\text {loc }}\left(\mathbb{R}^{n}\right)$ with finite quasinorms

$$
\begin{aligned}
\|f\|_{L W M_{p \theta, w}} & \equiv\|f\|_{L W M_{p \theta, w}\left(\mathbb{R}^{n}\right)}=\|w(r)\| f\left\|_{W L_{p}(B(0, r))}\right\|_{L_{\theta}(0, \infty)}, \\
\|f\|_{G W M_{p \theta, w}} & =\sup _{x \in \mathbb{R}^{n}}\|f(x+\cdot)\|_{L W M_{p \theta, w}},
\end{aligned}
$$

respectively, where for $p<\infty$,

$$
\|f\|_{W L_{p}(B(0, r))}=\sup _{t>0} t(\operatorname{meas}\{x \in B(0, r):|f(x)|>t\})^{1 / p} .
$$

If $p=\infty$, then $W L_{\infty} \equiv L_{\infty}$ and $L W M_{\infty \theta, w} \equiv L M_{\infty \theta, w}, G W M_{\infty \theta, w} \equiv$ $G M_{\infty \theta, w}$. 
Note that for any $0<p, \theta \leq \infty$,

$$
\|f\|_{L W M_{p \theta, w}} \leq\|f\|_{L M_{p \theta, w}}, \quad\|f\|_{G W M_{p \theta, w}} \leq\|f\|_{G M_{p \theta, w}}
$$

for all $f \in L M_{p \theta, w}$ and $f \in G M_{p \theta, w}$ respectively.

As in [2], [5] and [7] the proof of the boundedness of the maximal operator for $p=1$ is based on the inequality

$$
\text { meas }\{x \in B(0, r):(M f)(x)>t\} \leq \frac{c_{21}}{t} \int_{\mathbb{R}^{n}}|f(x)|\left(M \chi_{B(0, r)}\right)(x) d x,
$$

where $c_{21}>0$ is independent of $f \in L_{1}^{\text {loc }}\left(\mathbb{R}^{n}\right), t$ and $r$. This is a particular case of a more general inequality established by C. L. Fefferman and E. Stein [3]:

$$
\int_{\left\{x \in \mathbb{R}^{n}:(M f)(x)>t\right\}}|\varphi(x)| d x \leq \frac{c_{22}}{t} \int_{\mathbb{R}^{n}}|f(x)|(M \varphi)(x) d x,
$$

where $c_{22}>0$ is independent of $f, \varphi \in L_{1}^{\text {loc }}\left(\mathbb{R}^{n}\right)$.

Using inequality (38), the relation

$$
\|M f\|_{W L_{p}\left(\mathbb{R}^{n}\right)} \leq\|M f\|_{L_{p}\left(\mathbb{R}^{n}\right)}, \quad 0<p \leq \infty,
$$

and the properties of the maximal operator in local Morrey-type spaces established in the first part of the paper, we get the following corresponding properties of the maximal operator in local weak Morrey-type spaces:

Lemma 10. Let $1 \leq p<\infty$. Then there exists $c_{23}>0$ such that

$$
\|M f\|_{W L_{p}(B(0, r))} \leq c_{23}\left(r^{n} \int_{r}^{\infty}\left(\int_{B(0, t)}|f(x)|^{p} d x\right) \frac{d t}{t^{n+1}}\right)^{1 / p}
$$

for all $r>0$ and all $f \in L_{1}^{\mathrm{loc}}\left(\mathbb{R}^{n}\right)$.

Lemma 11. Let $1 \leq p<\infty, 0<\theta \leq \infty$. Then there exists $c_{24}>0$ such that

$$
\|M f\|_{L W M_{p \theta, w}} \leq c_{24}\|H g\|_{L_{\theta / p, v}(0, \infty)}^{1 / p}
$$

for all $f \in L_{p}^{\text {loc }}\left(\mathbb{R}^{n}\right)$, where $g$ and $v$ are given by (15), (16) respectively.

Corollary 5. Let $1 \leq p<\infty, 0<\theta \leq \infty$. Then

$$
\|M f\|_{G W M_{p \theta, w}} \leq c_{24} \sup _{x \in \mathbb{R}^{n}}\|H(g(x, \cdot))\|_{L_{\theta / p, v}(0, \infty)}^{1 / p}
$$

for all $f \in L_{p}^{\text {loc }}\left(\mathbb{R}^{n}\right)$, where $g(x, \cdot)$ is given by $(18)$.

Proof. We consider two cases:

1. If $1<p<\infty$, the assertion follows by the proof of Corollary 3 .

2. If $p=1$, then for all $x \in \mathbb{R}^{n}$, 


$$
\begin{aligned}
& \|(M f)(x+\cdot)\|_{W L_{1}(B(0, r))} \\
& =\sup _{t>0} t\left|\left\{z \in B(0, r): \sup _{r>0} \frac{1}{|B(x+z, r)|} \int_{B(x+z, r)}|f(y)| d y>t\right\}\right| \\
& =\sup _{t>0} t\left|\left\{z \in B(0, r): \sup _{r>0} \frac{1}{|B(z, r)|} \int_{B(z, r)}|f(x+y)| d y>t\right\}\right| \\
& =\|M(f(x+\cdot))\|_{W L_{1}(B(0, r))},
\end{aligned}
$$

hence we have

$$
\begin{aligned}
\|M f\|_{G W M_{1 \theta, w}} & =\sup _{x \in \mathbb{R}^{n}}\|w(r)\|(M f)(x+\cdot)\left\|_{W L_{1}(B(0, r))}\right\|_{L_{\theta}(0, \infty)} \\
& =\sup _{x \in \mathbb{R}^{n}}\|w(r)\| M(f(x+\cdot))\left\|_{W L_{1}(B(0, r))}\right\|_{L_{\theta}(0, \infty)} \\
& =\sup _{x \in \mathbb{R}^{n}}\|M(f(x+\cdot))\|_{L W M_{1 \theta, w}} \\
& \leq c_{24} \sup _{x \in \mathbb{R}^{n}}\left\|H\left(\int_{B\left(0, t^{-1 / n}\right)}|f(x+y)| d y\right)\right\|_{L_{\theta, v}(0, \infty)} \\
& =c_{24} \sup _{x \in \mathbb{R}^{n}}\|H(g(x, \cdot))\|_{L_{\theta, v}(0, \infty)} .
\end{aligned}
$$

TheOREM 5. Let $0<p_{2} \leq p_{1}<\infty, p_{1}>1,0<\theta_{1}, \theta_{2} \leq \infty, w_{1} \in$ $\Omega_{p_{1}, \theta_{1}}, w_{2} \in \Omega_{p_{2}, \theta_{2}}$. Assume that, for some $q>1$ satisfying $p_{2} \leq q \leq$ $p_{1}$, the operator $H$ is bounded from $L_{\theta_{1} / q, v_{1}}(0, \infty)$ to $L_{\theta_{2} / q, v_{2}}(0, \infty)$ on the cone of all non-negative functions $\varphi$ non-increasing on $(0, \infty)$ and satisfying $\lim _{t \rightarrow \infty} \varphi(t)=0$, where $v_{1}, v_{2}$ are defined by $(21),(22)$ respectively. Then the operator $M$ is bounded from $L M_{p \theta_{1}, w_{1}}$ to $L W M_{p \theta_{2}, w_{2}}$ and from $G M_{p \theta_{1}, w_{1}}$ to $G W M_{p \theta_{2}, w_{2}}$.

Theorem 6. Let $1 \leq p<\infty, 0<\theta_{2} \leq \infty, w_{1} \in \Omega_{p, \infty}, w_{2} \in \Omega_{p, \theta_{2}}$. Let also condition (29) be satisfied. Then the operator $M$ is bounded from $L M_{p \infty, w_{1}}$ to $L W M_{p \theta_{2}, w_{2}}$ and from $G M_{p \infty, w_{1}}$ to $G W M_{p \theta_{2}, w_{2}}$.

Lemma 12. Let $r, t>0$, and $0<p \leq \infty$. Then

$\left\|f_{t}\right\|_{W L_{p}(B(0, r))}=0, \quad 0<r<t, \quad\left\|f_{t}\right\|_{W L_{p}(B(0, r))} \leq c_{15} t^{n / p}, \quad r \geq t$.

Proof. The statement follows from Lemma 7 since for all measurable $G, \Omega \subset \mathbb{R}^{n}$,

$$
\left\|\chi_{G}\right\|_{W L_{p}(\Omega)}=|G \cap \Omega|^{1 / p}=\left\|\chi_{G}\right\|_{L_{p}(\Omega)},
$$

hence $\left\|f_{t}\right\|_{W L_{p}(B(0, r))}=\left\|f_{t}\right\|_{L_{p}(B(0, r))}$.

Lemma 13. For all $0<p \leq \infty$,

$$
\left\|M f_{t}\right\|_{W L_{p}(B(0, r))} \asymp\left(\frac{t}{r+t}\right)^{n \min \{1 / p, 1\}} r^{n / p} .
$$


Proof. By Lemma 8 we have

$$
\left\|M f_{t}\right\|_{W L_{p}(B(0, r))} \asymp t^{n}\left\|\left(\frac{1}{|x|+t}\right)^{n}\right\|_{W L_{p}(B(0, r))} .
$$

Furthermore,

$$
\begin{aligned}
& \left\|\left(\frac{1}{|x|+t}\right)^{n}\right\|_{W} L_{p}(B(0, r)) \\
& =\sup _{\tau>0} \tau\left|B(0, r) \cap B\left(0, \tau^{-1 / n}-t\right)\right|^{1 / p} \\
& =v_{n} \sup _{0<\tau<t^{-n}} \tau\left(\min \left\{r, \tau^{-1 / n}-t\right\}\right)^{n / p} \\
& =v_{n} \max \left\{\sup _{0<\tau \leq(t+r)^{-n}} \tau r^{n / p}, \sup _{(t+r)^{-n}<\tau<t^{-n}} \tau\left(\tau^{-1 / n}-t\right)^{n / p}\right\} \\
& =v_{n} \max \left\{(t+r)^{-n} r^{n / p}, \sup _{(t+r)^{-n}<\tau<t^{-n}} \tau\left(\tau^{-1 / n}-t\right)^{n / p}\right\} \\
& =\sup _{(t+r)^{-n} \leq \tau<t^{-n}} \tau\left(\tau^{-1 / n}-t\right)^{n / p} .
\end{aligned}
$$

If $0<p \leq 1$, then the function $\phi(\tau)=\tau\left(\tau^{-1 / n}-t\right)^{n / p}$ decreases on $\left[(t+r)^{-n}, t^{-n}\right)$, therefore

$$
\sup _{(t+r)^{-n} \leq \tau<t^{-n}} \tau\left(\tau^{-1 / n}-t\right)^{n / p}=\frac{r^{n / p}}{(t+r)^{n}} .
$$

If $p>1$, then for $t \geq(p-1) r, \varphi$ also decreases on $\left[(t+r)^{-n}, t^{-n}\right)$ and for $t<(p-1) r$ the supremum is attained at $\tau=\left(\frac{p-1}{p t}\right)^{n}$. Hence

$$
\begin{aligned}
\sup _{(t+r)^{-n} \leq \tau<t^{-n}} \tau\left(\tau^{-1 / n}-t\right)^{n / p} & =c_{25} \begin{cases}\frac{r^{n / p}}{(t+r)^{n}}, & t \geq(p-1) r, \\
t^{n / p-n}, & t<(p-1) r,\end{cases} \\
& \asymp\left(\frac{r t}{t+r}\right)^{n / p} t^{-n},
\end{aligned}
$$

where $c_{25}>0$ depends only on $p$ and $n$. Therefore the statement follows.

TheOREM 7. Let $1<p<\infty, 0<\theta_{1}, \theta_{2} \leq \infty, w_{1} \in \Omega_{p, \theta_{1}}, w_{2} \in \Omega_{p, \theta_{2}}$.

- If $\theta_{1} \leq \theta_{2}, \theta_{1} \leq p$ and inequality (35) is satisfied, then $M$ is bounded from $L M_{p \theta_{1}, w_{1}}$ to $L W M_{p \theta_{2}, w_{2}}$ and from $G M_{p \theta_{1}, w_{1}}$ to $G W M_{p \theta_{2}, w_{2}}$.

- For any $0<\theta_{1}, \theta_{2} \leq \infty$ condition (35) is necessary for the boundedness of $M$ from $L M_{p \theta_{1}, w_{1}}$ to $L W M_{p \theta_{2}, w_{2}}$.

- In particular, if $\theta_{1} \leq \theta_{2}, \theta_{1} \leq p$, then condition (35) is necessary and sufficient for the boundedness of $M$ from $L M_{p \theta_{1}, w_{1}}$ to $L W M_{p \theta_{2}, w_{2}}$. 
Proof. Sufficiency follows from Theorem 5 as in the proof of Theorem 4. The proof of necessity is also essentially the same as in the proof of Theorem 4, with Lemma 9 replaced by Lemma 13.

REMARK 4. When defining global Morrey-type spaces, one might consider a weight function $w$ depending not only on $r>0$, but also on $x \in \mathbb{R}^{n}$, and consider the space of all functions $f \in L_{p}^{\text {loc }}\left(\mathbb{R}^{n}\right)$ for which

$$
\|\| w(x, r)\|f\|_{L_{p}(B(x, r))}\left\|_{L_{\theta}(0, \infty)}\right\|_{L_{\infty}\left(\mathbb{R}^{n}\right)}<\infty .
$$

For $\theta=\infty$ such quasinorms were considered in [7]. Moreover, it is also reasonable to replace $L_{\infty}\left(\mathbb{R}^{n}\right)$ by $L_{\eta}\left(\mathbb{R}^{n}\right)$, where $0<\eta \leq \infty$, thus assuming that

$$
\|f\|_{G M_{p \theta \eta, w}}=\|\| w(x, r)\|f\|_{L_{p}(B(x, r))}\left\|_{L_{\theta}(0, \infty)}\right\|_{L_{\eta}\left(\mathbb{R}^{n}\right)}<\infty .
$$

If in Theorem 2 formulas (21) and (22) are replaced by

$$
\begin{aligned}
& v_{1}(x, r)=\left(w_{1}\left(x, r^{-1 / n}\right) r^{1 / q-1 / p_{1}-(1+1 / n) 1 / \theta_{1}}\right)^{q}, \\
& v_{2}(x, r)=\left(w_{2}\left(x, r^{-1 / n}\right) r^{-1 / p_{2}-(1+1 / n) 1 / \theta_{2}}\right)^{q}
\end{aligned}
$$

and

$$
\sup _{x \in \mathbb{R}^{n}}\|H\|_{L_{\theta_{1} / q, v_{1}(x, r)}(0, \infty) \cap C \rightarrow L_{\theta_{2} / q, v_{2}(x, r)}(0, \infty) \cap C}<\infty,
$$

where $C$ is the cone of all non-negative functions $\varphi$ non-increasing on $(0, \infty)$ and satisfying $\lim _{t \rightarrow \infty} \varphi(t)=0$, then the maximal operator $M$ is also bounded from $G M_{p_{1} \theta_{1} \eta, w_{1}}$ to $G M_{p_{2} \theta_{2} \eta, w_{2}}$. Similar remarks refer to all other inequalities of the paper involving global Morrey-type spaces or global weak Morrey-type spaces.

A brief exposition of the results of this paper, without proofs, is given in $[1]$.

\section{References}

[1] V. I. Burenkov and H. V. Guliyev, Necessary and sufficient conditions for boundedness of the maximal operator in local Morrey-type spaces, Dokl. Ross. Akad. Nauk 391 (2003), 591-594 (in Russian).

[2] F. Chiarenza and M. Frasca, Morrey spaces and Hardy-Littlewood maximal function, Rend. Mat. 7 (1987), 273-279.

[3] C. L. Fefferman and E. M. Stein, Some maximal inequalities, Amer. J. Math. 93 (1971), 107-115.

[4] G. Lu, Embedding theorems on Campanato-Morrey spaces for vector fields and applications, C. R. Acad. Sci. Paris 320 (1995), 429-434.

[5] T. Mizuhara, Boundedness of some classical operators on generalized Morrey spaces, in: Harmonic Analysis (S. Igari, ed.), ICM 90 Satellite Proceedings, Springer, Tokyo, 1991, 183-189. 
[6] C. B. Morrey, On the solutions of quasi-linear elliptic partial differential equations, Trans. Amer. Math. Soc. 43 (1938), 126-166.

[7] E. Nakai, Hardy-Littlewood maximal operator, singular integral operators and Riesz potentials on generalized Morrey spaces, Math. Nachr. 166 (1994), 95-103.

[8] S. M. Nikol'skiǔ, Approximation of Functions of Several Variables and Embedding Theorems, Nauka, Moscow, 1969 (in Rusian); English translation, Springer, 1975.

[9] J. Peetre, On the theory of $L^{p, \lambda}$ spaces, J. Funct. Anal. 4 (1969), 71-87.

[10] E. Sawyer, Two weight norm inequalities for certain maximal and integral operators, in: Harmonic Analysis (Minneapolis, MN, 1981), Lecture Notes in Math. 908, Springer, 1982, 102-127.

[11] S. Spanne, Sur l'interpolation entre les espaces $\mathcal{L}_{k}^{p, \Phi}$, Ann. Scuola Norm. Sup. Pisa 20 (1966), 625-648.

[12] V. D. Stepanov, Weighted Hardy inequalities for increasing functions, Canad. J. Math. 45 (1993), 104-116.

[13] - , The weighted Hardy's inequalities for nonincreasing functions, Trans. Amer. Math. Soc. 338 (1993), 173-186.

School of Mathematics

Cardiff University

PO Box 926

Cardiff, CF24 4YH

United Kingdom

E-mail: Burenkov@cardiff.ac.uk

Guliyev@Huseyn.com

Received February 10, 2003

Revised version November 25, 2003 